\title{
Caractérisation technologique de souches pures de bactéries propioniques : test en minifabrication de fromages à pâte cuite
}

\author{
R Richoux, JR Kerjean \\ Institut technique du gruyère, BP 6224, 35062 Rennes Cedex, France
}

(Reçu le 3 mars 1994 ; accepté le 29 septembre 1994)

\begin{abstract}
Résumé - L'aptitude technologique de 18 souches de Propionibacterium freudenreichii et d'une souche de $P$ thoenii a été déterminée en technologie emmental. Soixante-six minifabrications de fromages à pâte cuite de type emmental ont été effectuées à partir de lait microfiltré. La préparation des ferments propioniques et la fabrication des fromages étaient standardisées. Pendant l'affinage en cave chaude, des mesures hebdomadaires du volume des fromages et des analyses bimensuelles de teneur en acides gras volatils et en lactates ont permis de classer statistiquement les souches en 5 groupes. L'évolution du volume et de la teneur en acide propionique sont des indices complémentaires, mais aussi les indices les plus discriminants. Les souches des 4 premiers groupes présentent une aptitude technologique certaine, avec des différences notables de temps de latence, de vitesses de fermentation et de synchronisme des productions de propionate et de gaz carbonique. La fermentation de ces souches semble s'achever après 14 à 28 jours. Les souches du dernier groupe apparaissent inadaptées en technologie emmental : absence de croissance, croissance lente ou production d'acides gras volatils sans dégagement gazeux massif.
\end{abstract}

Propionibacterium / emmental / acide gras volatil / $\mathrm{CO}_{2} /$ affinage

Summary - Technological properties of pure propionibacteria strains: test in small scale Swiss-type cheese. Eighteen strains of Propionibacterium freudenreichii and one strain of $\mathrm{P}$ thoenii were tested for their ability to produce volatile fatty acids and gas in small scale Swiss-type cheese. Microfiltrated milk and standardized propionic strains were used. Sixty-six mini-cheeses were made according to a standardized classical emmental cheesemaking process. During the hot room ripening, weekly determination of cheese volume and bimonthly analysis of volatile fatty acids and lactate contents permitted to distinguish five groups of strains. Propionate content and change in cheese volume were the most discriminant parameters. Four groups of strains showed different technological properties, where propionic fermentation seemed to be achieved after 14 to 28 days. Differences appeared in latent period, fermentation velocity and synchronism of gas and propionate productions. The strains of the last group were unsuitable for Swiss-type cheese technology. Some of them did not grow in the cheeses, some grew very slowly and others produced volatile fatty acids but not $\mathrm{CO}_{2}$ on a great scale. Weekly 
determinations of volatile fatty acids and lactate contents permitted a better characterization of the more precocious strains. As a general rule, the technological properties also reflected differences in growth and metabolic activities.

Propionibacterium / Swiss-type cheese / volatile fatty acid / $\mathrm{CO}_{2} /$ ripening

\section{INTRODUCTION}

La participation des bactéries propioniques laitières à l'affinage des fromages à pâte cuite de type emmental est connue depuis longtemps (Sherman, 1921 ; Reinbold, 1972 ; Langsrud et Reinbold, 1973; Steffen et al, 1993). Elles fermentent les lactates produits par les bactéries lactiques, principalement en dioxyde de carbone, acétate, propionate et succinate (Crow et Turner, 1986 ; Sebastiani et Bader, 1992). Ces composés participent à l'ouverture, au goût et à l'arôme caractéristiques de l'emmental.

De récentes études ont mis en évidence d'autres aspects intéressants, du point de vue fromager, du métabolisme des bactéries propioniques : activité estérasique et lipolytique (Dupuis et Boyaval, 1993 ; Dupuis et al, 1993), peptidasique (Sahlstrom et al, 1989 ; El Soda et al, 1992), potentiel autolytique (Lemée et al, 1994). L'existence de bactériophages infectant Propionibacterium freudenreichii vient d'être démontrée par Gautier et al (1992b). Les interactions microbiennes entre les propionibactéries et les bactéries lactiques revêtent également une grande importance technologique (Hettinga et Reinbold, 1972 ; Perez Chaia et al, 1987 ; Zehntner, 1993 ; Sollberger, 1994).

Cependant, les progrès en matière d'hygiène de production du lait et la mise en œuvre de techniques puissantes d'épuration du lait (bactofugation, microfiltration) ont provoqué une chute importante de la flore propionique sauvage dans les laits de fabrication. Un réensemencement apparaiit donc aujourd'hui indispensable. Pour cela, il est nécessaire de caractériser les souches de bactéries propioniques en termes d'apti- tude technologique. Jusqu'à présent, les travaux dans ce sens n'ont concerné que le métabolisme carboné, l'avancement des études sur les autres paramètres ne permettant pas, à l'heure actuelle, de définir et de mesurer des critères de sélection pertinents. Ces aspects n'en sont pas moins importants et devront être à plus ou moins long terme intégrés dans la démarche de caractérisation technologique.

La plupart de ces études de caractérisation consistent en des fabrications fromagères en technologie d'emmental ou de gruyère (Vassal et Auclair, 1974 ; Fryer et Peberdy, 1977 ; Hollywood et Doelle, 1984 ; Crow et Turner, 1986 ; Pérréard, 1992 ; Baer et al,1993). Malheureusement, ces études mettent en œuvre un nombre limité de souches (1 à 7 ) et des laits incomplètement épurés (au mieux par bactofugation ou crèmage : Pếrréard, 1992) présentant une flore résiduelle, propionique ou non, qui risque d'interférer avec les réensemencements lactique et propionique. De plus, la plupart d'entre elles appréhendent plus la croissance des bactéries propioniques que les cinétiques globales d'évolution des fromages.

Plusieurs auteurs ont entrepris des tests de longue durée en fermenteur sur milieu complexe (Crow et Turner, 1986 ; Sebastiani et Bader, 1992 ; Trossat, 1992) à pH régulé ou non, avec une cinétique d'évolution des métabolites : lactate, $\mathrm{CO}_{2}$, acétate et propionate, et évolution du succinate et de l'aspartate pour Crow et Turner (1986) ou Sebastiani et Bader (1992). Ces derniers préconisent comme critère de sélection d'importantes vitesses de consommation du lactate et de l'aspartate et de production 
de succinate. Ces méthodes sont cependant lourdes, coûteuses et effectuées sur un milieu différant du fromage.

La minifabrication expérimentale, développée par l'Institut technique du gruyère (ITG) depuis 1987 (Buisson et al, 1987 ; Kerjean, 1991) peut s'avérer un outil de test intéressant dans le domaine de la caractérisation de l'aptitude technologique des bactéries propioniques, c'est-à-dire leur aptitude à produire du gaz carbonique et d'autres métabolites en conditions technologiques constantes. Par sa technologie réaliste, éprouvée, standardisée et reproductible, elle constitue une étape intermédiaire entre les tests de laboratoire (Trossat, 1992) et la fromagerie expérimentale (Pérréard, 1992). En outre, le developpement des techniques d'épuration microbienne par microfiltration tangentielle (Trouvé et al, 1991) et les progrès en matière de purification des souches de propionibactéries autorisent des réensemencements maîtrisés de laits «ultrapropres".

L'objet de cette étude consistait donc à déterminer si la minifabrication peut être un outil pertinent, discriminant, répétable (voire économique) pour appréhender l'aptitude technologique des souches de bactéries propioniques.

\section{MATÉRIELS ET MÉTHODES}

\section{Souches de bactéries propioniques}

Dix-huit souches pures de Propionibacterium freudenreichii, caractérisées sur galerie API $50 \mathrm{CH}$ (Biomérieux, France) et par électrophorèse en champs pulsés (Gautier et al, 1992a), ont été sélectionnées dans la collection privée emmental du SIGF (Syndicat Interprofessionnel du gruyère français, Paris, France). Elles sont issues d'emmentals de bonne qualité et sont numérotées de 1 à 18 . Une souche de $P$ thoenii CNRZ 85R (Centre national de recherche zootechnique,
Jouy-en-Josas, France) a également été testée. Ces souches apparaissent différentes au regard de leur profil biochimique et de leur profil de restriction de l'ADN.

\section{Préparation des ferments propioniques}

Pour obtenir des ensemencements réguliers du point de vue quantitatif et qualitatif (état physiologique des bactéries) la préparation des ferments propioniques était standardisée. Le lot de départ consistait en des cultures sur milieu BYC lactate (Naud et al, 1988) additionné de $15 \%$ de glycérol (Prolabo, Paris, France) congelèes à $-18^{\circ} \mathrm{C}$ en tubes cryogéniques. Pour chaque fabrication fromagère, un échantillon du lot de départ était décongelé ; $10 \mathrm{ml}$ de bouillon BYC lactate étaient ensemencés à $5 \%(\mathrm{v} / \mathrm{v})$ et incubés à $30^{\circ} \mathrm{C}$ jusqu'à l'obtention d'une densité optique $\left(D_{650 n m}\right.$ ) de 1 (spectrophotomètre Beckman DU 7400, Fullerton, Californie, États-Unis). Cette étape durait en moyenne $24 \mathrm{~h}$. Après un examen microscopique, un repiquage à $2 \%(v / v)$ dans $100 \mathrm{ml}$ de bouillon BYC lactate était pratiqué. Ce ferment propionique était ensuite cultivé jusqu'à utilisation, en moyenne $36 \mathrm{~h}$. Le temps de génération n'étant pas identique pour les différentes souches, la durée d'incubation était modulée de manière à obtenir une $\mathrm{DO}_{650 \mathrm{~nm}}$ voisine de 1 au moment de l'utilisation et d'éviter ainsi toute altération bactérienne par un report au froid.

\section{Ensemencement des laits}

Avant l'ensemencement, un examen microscopique était pratiqué pour contrôler le ferment propionique. En fonction de la densité optique obtenue, la quantité de ferment inoculée en cuve de fromagerie était modulée de manière à pratiquer un ensemencement de l'ordre de $510^{5} \mathrm{ufc} / \mathrm{ml}$ de lait. Faute de milieu de dénombrement suffisamment sélectif sur lait, le contrôle de l'ensemencement était réalisé par le dénombrement de la population des ferments sur gélose BYC lactate à $30^{\circ} \mathrm{C}$ sous anaérobiose (Anaérocult A, Merck, Darmstadt, Allemagne). Le niveau d'ensemencement du lait était ensuite déterminé par calcul : (population des ferments) $\times$ (volume inoculé) / (volume de lait). 


\section{Minifabrications}

Des matières premières prétraitées à l'atelier Compagnie laitière européenne (Montauban de Bretagne, France) étaient utilisées. Du lait écrémé et de la crème thermisés issus de lait de grand mélange étaient prélevés sur l'installation de stan- dardisation de lait de fabrication emmental. Le lait était réchauffé à $50^{\circ} \mathrm{C}$, puis épuré par microfiltration, comme décrit par Trouvé et al (1991). L'installation pilote de type Bactocatch (MFS1, Alfa Laval, Les-Clayes-sous-Bois, France) était équipée d'une membrane céramique Membralox (Société des céramiques techniques, Tarbes, France) monocorps de $0,2 \mathrm{~m}^{2}$ de surface filtrante

Tableau I. Schéma de fabrication des mini-fromages.

Cheesemaking process.

\section{Spécification}

$10,3 \mathrm{~kg}$ de lait

$0,07 \mathrm{~g} / \mathrm{l}$

$7 \mathrm{U} / 10001$

$4 U / 1000$ ।

$4 \mathrm{U} / 1000$ I

Ferments lactiques $\mathrm{LH}$

Ferments propioniques

Coagulation

$\mathrm{pH}$ emprésurage

Dose présure $(800 \mathrm{mg} / \mathrm{l})$

Prise

Durcissement

Décaillage

Brassage avant feu

Chauffage

Brassage après feu

Soutirage

Pressage

Acidification

Refroidissement

Salage

Saumure saturée

Réessuyage

Affinage

$20 \mathrm{ml} / 100 \mathrm{I}$

$80 \mathrm{~g} / \mathrm{cm}^{2}$

$160 \mathrm{~g} / \mathrm{cm}^{2}$
Température

Durée

$5^{\circ} \mathrm{C} \longrightarrow 32^{\circ} \mathrm{C}$

$20 \mathrm{~min}$

$32^{\circ} \mathrm{C}$

$60 \mathrm{~min}$

$32^{\circ} \mathrm{C}$

$27 \mathrm{~min}$

600 U du gélographe

$20 \mathrm{~min}$

$7 \mathrm{~min}$

allure standardisée

$32^{\circ} \mathrm{C}$

$4 \min$

$32^{\circ} \mathrm{C}$

$15 \mathrm{~min}$

$32^{\circ} \mathrm{C} \longrightarrow 53,5^{\circ} \mathrm{C}$

$33 \mathrm{~min}$

$53,5^{\circ} \mathrm{C}$

$45 \mathrm{~min}$

$53,5^{\circ} \mathrm{C}$

$48^{\circ} \mathrm{C}$

$48^{\circ} \mathrm{C}$

$30 \mathrm{~min}$

$5,5 \mathrm{~h}$

$36^{\circ} \mathrm{C}$

$15,5 \mathrm{~h}$

$36^{\circ} \mathrm{C} \longrightarrow 7^{\circ} \mathrm{C}$

$5 \mathrm{~h}$

$7^{\circ} \mathrm{C}$

$3,5 \mathrm{~h}$

$7^{\circ} \mathrm{C}$

$48 \mathrm{~h}$

$11^{\circ} \mathrm{C}$

$24^{\circ} \mathrm{C}$
$21 \mathrm{j}$

$28 \mathrm{j}$ 
et d'un diamètre de pores moyen de $1,4 \mu \mathrm{m}$. Seul le perméat était collecté et refroidi à $4^{\circ} \mathrm{C}$, le faible volume de lait mis en œuvre ne permettant pas une récupération et un traitement rigoureux du rétentat.

Les minifabrications suivaient le procédé standardisé habituellement mis en œuvre à l'ITG (Buisson et al, 1987; Kerjean 1991) et résumé dans le tableau I. Les ferments lactiques lyophilisés concentrés à ensemencement direct provenaient de la société Texel (Dangé Saint Romain, France) :

- lactocoques MA 014, lots $90205 \mathrm{C}$ et 91 193A : Lactococcus lactis subsp lactis et Lactococcus lactis subsp cremoris ;

- streptocoques thermophiles TA 060 lot 90 196A et TA 061 lot 92 027A : Streptococcus salivarius subsp thermophilus ;

- lactobacilles thermophiles LH 100 lots $90143 A$ et 91 193A : Lactobacillus helveticus et Lactobacillus delbrueckii subsp lactis.

Les cuves étaient soutirées dans des moules de $9,2 \mathrm{~cm}$ de diamètre. Après $30 \mathrm{~min}$ de pressage, le caillé était divisé en 2 fromages jumeaux présentant la même hauteur que les minifromages classiquement fabriqués à l'ITG (Buisson et al, 1987). Après le ressuyage suivant le saumurage, l'un des 2 fromages jumeaux était découpé en 4 pour le suivi physico-chimique en cours d'affinage. Les 4 parts et l'autre fromage jumeau étaient paraffinés (Cire W289, Sergent Prolac, Surgères, France) et affinés 21 jours à $11^{\circ} \mathrm{C}$ puis 28 jours à $24^{\circ} \mathrm{C}$, suivant en cela un cycle d'affinage "typique" de l'emmental.

\section{Suivi analytique}

La composition chimique du lait en vrac était contrôlée après standardisation par un dosage par absorption infra-rouge (Dairylab 2, Multispec, York, Royaume-Uni). Un dosage de la matière grasse du lait (FILIDF, 1991b) en cuve permettait de vérifier l'homogénéité du prélèvement du lait pour la fabrication. Au cours de la dernière fabrication, soit après $60 \mathrm{~h}$ de stockage à $4^{\circ} \mathrm{C}$, un contrôle microbiologique était effectué, qui correspond au niveau maximal de contamination des laits :

- flore mésophile aérobie revivifiable (FMAR): norme FIL 100 (FIL/IDF, 1991a) sur gélose PCA (Difco, États-Unis);
- flore présumée propionique résiduelle : dénombrement sur gélose $\mathrm{BYC}$ lactate à $30^{\circ} \mathrm{C}$ pendant 8 jours en anaérobiose, comptage des grosses colonies lenticulaires de couleur beige caractéristiques ;

- spores de Clostridia fermentant les lactates (CNERNA, 1986): ensemencement $10 \mathrm{ml}$ (bouillon double concentration), $1 \mathrm{ml}, 0,1 \mathrm{ml}$.

Les contrôles de fabrication classiques étaient effectués : suivi de coagulation au gélograph (Gel Instrument, Thalwil, Suisse) et d'acidification par pH métrie (Tacussel pHN 850, SOLEA, Villeurbanne, France). Pour vérifier le bon déroulement du travail en cuve, les teneurs en matière grasse du lactosérum au soutirage (FILIDF, 1991b) et du caillé après 30 min de pressage (Heiss, 1961) étaient mesurées par dosage acido-butyrométrique. L'extrait sec du caillé (FILIDF, 1982) à 30 min était également contrôlé.

Les portions prédécoupées étaient utilisées pour les analyses en cours d'affinage à 10 jours de cave froide, en sortie de cave froide ( 21 jours), après 14 jours de cave chaude ( 35 jours) et en sortie de cave chaude (49 jours). Pour certaines expérimentations, un suivi hebdomadaire en cave chaude a été réalisé (souches 11 à 18). Ces analyses hebdomadaires permettent le calcul de temps de latence et de vitesses de fermentation approximatifs. Le temps de latence correspond à l'intervalle de 7 jours précédant l'apparition d'une différence significative pour chacun des traceurs : acétate, propionate, lactate. Arbitrairement, la durée de fermentation est déterminée comme le temps écoulé entre le dernier prélèvement sans différence significative et la date d'obtention d'un palier de composition. La vitesse est alors calculée par le rapport des différences de composition à ces dates, divisé par la durée de fermentation. En prenant en compte la composition au dernier prélèvement non significatif, et non la composition à l'origine, on élimine l'évolution pendant la "phase d'adaptation" et on considère, arbitrairement, que l'évolution est linéaire.

Les échantillons étaient emballés dans du papier d'aluminium, congelés à $-18^{\circ} \mathrm{C}$ et acheminés au laboratoire central de l'ITG (Rioz, France) pour analyse dans le mois suivant. Cette pratique ne modifie pas les résultats des dosages (Collomb et al, 1992). Les acides gras volatils (Berdagué, 1986), les lactates $L$ et $D$ (méthode enzymatique, Boehringer, Mannheim, Allemagne), l'ammoniaque (Courroye et al, 1989) et les groupements -NH2 libres (Samples et al, 1984) étaient dosés. 
En fin d'affinage, le fromage jumeau subissait les mêmes analyses afin de vérifier l'équivalence des fermentations dans les portions prédécoupées et dans le fromage entier. Des contrôles classiques de composition des fromages étaient pratiqués de manière à valider les fabrications : extrait sec total, matières grasses, chlorures par dosage potentiométrique (Chloruremètre Corning 426, Halstead, Royaume-Uni). Différents indices de protéolyse étaient également mesurés : azote soluble à pH 4,6 , azote soluble dans le TCA à 12\% (Courroye et al, 1989), électrophorèse des caséines (Collin et al, 1987).

Le volume du fromage jumeau était mesuré par immersion dans l'eau après 10 jours, en sortie de cave froide, puis toutes les semaines de cave chaude. Une jarre, munie d'un couvercle percé assurant une bonne évacuation de l'air, était remplie d'eau à la température du fromage, essuyée et pesée. Le fromage, préalablement pesé, était introduit dans la jarre. Cette dernière était complétée d'eau, essuyée et pesée. Le volume brut était alors calculé :

Volume brut $=$ [masse $($ fromage $)+$ masse (jarre + eau) - masse (jarre + fromage + eau)] $x$ volume massique de l'eau

En fin d'affinage, le volume de la paraffine était mesuré et retranché au volume brut. Les résultats étaient exprimés en indice, soit le volume net divisé par le volume net initial. Des essais préliminaires ont montré que le coefficient de variation des mesures de volume net avoisine $0,4 \%$. Le faible volume des fromages ne permettait pas de suivi bactériologique des fromages.

\section{Protocole expérimental}

Trois répétitions par souche de Propionibacterium étaient confrontées à 9 témoins, c'est-à-dire des fromages fabriqués sans ensemencement propionique. Un témoin était réalisé par semaine de fabrication, après $60 \mathrm{~h}$ de stockage, pour permettre en parallèle un contrôle technologique du lait (absence de dérive technologique après le report du lait).

Connaissant la moyenne et l'écart type habituels des concentrations en propionate des fromages (Buisson et al, 1987), ce nombre de répétitions permet a priori de mettre en évidence des différences de $200 \mathrm{mg} / 100 \mathrm{~g}$ entre traitements avec un risque de première espèce de 0,10 (test bilatéral) et une puissance de $60 \%$ (Philippeau, 1984).

\section{Traitement des résultats}

Les résultats étaient traités par analyse de variance à un facteur (souche ensemencée) au seuil de 0,05 sur logiciel STAT-ITCF (Gouet et Philippeau, 1989). Un test de Dunett discriminait les traitements identiques aux témoins de ceux supérieurs. Le test de Newman et Keuls classait ensuite ces derniers en groupes homogènes.

L'équivalence de composition des portions prédécoupées et des fromages jumeaux affinés était vérifiée par une comparaison de moyenne d'échantillons appariés : test de Student à 0,05.

\section{RÉSULTATS}

\section{Ensemencement en bactéries propioniques}

L'analyse de variance des niveaux d'ensemencement après transformation logarithmique ne montre pas de différence significative $(P>0,05)$. La moyenne générale s'élève à $3,2510^{5} \mathrm{ufc} / \mathrm{ml}(5,5 \mathrm{log})$, l'écart type à $0,243 \mathrm{log}$. Globalement, $95 \%$ des ensemencements étaient donc compris entre $10^{5}$ et $10^{6} \mathrm{ufc} / \mathrm{ml}$.

\section{Préparation du lait}

Les laits de départ contenaient $28,4 \mathrm{~g} / \mathrm{kg}$ $( \pm 1,96 \times 0,02 \mathrm{~g})$ de matières grasses, 31,7 $\mathrm{g} / \mathrm{kg}( \pm 1,96 \times 0,68 \mathrm{~g})$ de matières azotées, $47,7 \mathrm{~g} / \mathrm{kg}( \pm 1,96 \times 0,63 \mathrm{~g})$ de lactose et $111,1 \mathrm{~g} / \mathrm{kg}( \pm 1,96 \times 0,99 \mathrm{~g})$ d'extrait sec total. Après $60 \mathrm{~h}$ de stockage, la flore mésophile aérobie revivifiable s'élevait en moyenne à $190 \mathrm{ufc} / \mathrm{ml}$ (44 à $615 \mathrm{ufc} / \mathrm{ml}$ ). La flore présumée propionique restait inférieure à $5 \mathrm{ufc} / \mathrm{ml}$, la contamination sporulée butyrique à 20 spores par litre. 


\section{Fabrications fromagères}

Le tableau II présente les paramètres de fabrication et la composition des fromages affinés. Ces résultats sont en bon accord avec ceux de Buisson et al (1987) et Kerjean (1991). La légère diminution de l'aptitude à la coagulation (temps de durcissement) et à l'égouttage (humidité sur extrait sec dégraissé à 30 min de pressage) du lait microfiltré avait déjà été observée par Riberolles et Quiblier (1991). Aucune différence significative entre traitements n'apparaît $(P>0,05)$.

\section{Fermentations}

La composition des fromages évoluant très peu à $11^{\circ} \mathrm{C}$, le prélèvement effectué en milieu de cave froide a été supprimé pour l'étude des souches 11 à 18 . En effet, le volume des fromages et leur teneur en propionate augmentent en moyenne respectivement de $0,1 \%$ et $6 \mathrm{mg} / 100 \mathrm{~g}$, la teneur en acétate reste constante et la concentration en lactates chute de $16 \mathrm{mg} / 100 \mathrm{~g}$. De même, l'analyse statistique n'a pas montré de différence significative entre les portions et les fromages jumeaux (différence

Tableau II. Paramètres de fabrication et composition des mini-fromages affinés $(n=66)$. Cheesemaking results and ripened cheese composition.

\section{Moyenne Écart type}

\section{Acidification}

$\begin{array}{lll}\mathrm{pH} \text { lait } & 6,68 & 0,015 \\ \mathrm{pH} \text { emprésurage } & 6,63 & 0,016 \\ \mathrm{pH} \text { soutirage } & 6,41 & 0,022 \\ \mathrm{pH} 2 \mathrm{~h} & 6,18 & 0,078 \\ \mathrm{pH} 4 \mathrm{~h} & 5,57 & 0,072 \\ \mathrm{pH} 6 \mathrm{~h} & 5,47 & 0,041 \\ \mathrm{pH} 20 \mathrm{~h} & 5,17 & 0,047\end{array}$

Coagulation, égouttage

$\begin{array}{lcl}\text { Temps de prise }(\mathrm{min}) & 20,26 & 0,63 \\ \text { Temps de durcissement }(\mathrm{min}) & 7,4 & 0,51 \\ \text { MG lactosérum }(\mathrm{g} / \mathrm{l}) & 4,57 & 0,58 \\ \text { EST } 30 \mathrm{~min}(\mathrm{~g} / 100 \mathrm{~g}) & 58,18 & 0,54 \\ \text { ESD } 30 \mathrm{~min}(\mathrm{~g} / 100 \mathrm{~g}) & 31,02 & 0,78 \\ \text { Humidité/ESD } 30 \mathrm{~min} & 1,335 & 0,033\end{array}$

Fromages affinés

$\begin{array}{lcc}\mathrm{EST}(\mathrm{g} / 100 \mathrm{~g}) & 63,28 & 0,76 \\ \mathrm{Gras} / \mathrm{sec}(\%) & 46,03 & 1,33 \\ \text { Humidité/ESD } & 1,08 & 0,039 \\ \mathrm{NaCl}(\mathrm{g} / 100 \mathrm{~g}) & 0,36 & 0,047 \\ -\mathrm{NH}_{2} \text { libres (meq Gly/g) } & 220 & 43 \\ \mathrm{NH}_{4}{ }^{+}(\mathrm{mg} / 100 \mathrm{~g}) & 87,9 & 25,6\end{array}$


moyenne portion/fromage : $-27,6 \mathrm{mg} / 100$ $\mathrm{g}$ d'acétate, $-14,6 \mathrm{mg} / 100 \mathrm{~g}$ de propionate et $+12,5 \mathrm{mg} / 100 \mathrm{~g}$ de lactate), d'où l'abandon du double prélèvement en fin d'affinage. Ces modifications ont permis d'effectuer une cinétique hebdomadaire de fermentation en cave chaude.

L'interprétation globale a été effectuée sur la base des analyses bimensuelles en cave chaude. Les analyses hebdomadaires effectuées sur les fromages ensemencés avec les souches 11 à 18 viennent en complément : elles précisent simplement le comportement de ces souches.

\section{Volume des fromages}

La figure 1 montre l'évolution du volume des fromages pendant l'affinage en cave chaude. Les moyennes par groupe de souches de comportement statistiquement identique $(P>0,05)$ y sont représentées. Par souci de lisibilité, le graphique montre un plateau au niveau des valeurs maximales enregistrées. En effet, la rupture de la paraffine sous l'effet de la pression a entraîné des fuites de gaz se traduisant par une chute de volume après la phase d'expansion et des ouvertures généralement retombées. Une appréciation qualitative ou quantitative de l'ouverture en fin d'affinage n'a donc pas été possible. Néanmoins, l'analyse statistique dégage 4 types de comportements. Six souches $(5,6,8,10,17,18)$ produisent massivement du gaz carbonique après 14 jours de cave chaude. Huit souches $(2,3,4,7,9,14,15,16)$ font gonfler les minifromages à partir de 21 jours à $24^{\circ} \mathrm{C}$, tandis que la souche 12 agit tardivement après 28 jours. Quatre souches ( 1 , 11, 13, CNRZ 85R) ne se différencient pas des témoins en 28 jours de cave chaude, tout en provoquant une évolution du volume plus ou moins marquée. Ainsi, les souches 11 et 13 génèrent une très légère ouverture des fromages, tandis que les témoins restent totalement aveugles.

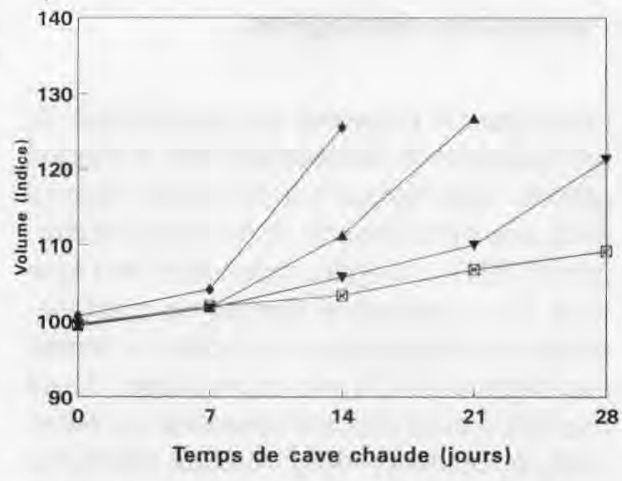

Fig 1. Évolution du volume des fromages pendant l'affinage en cave chaude. - - souches 5 ,

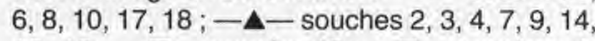
15,$16 ;-\nabla$ - souche $12 ;-\square$ - souches 1 , 11,13, CNRZ 85R, Tèmoins.

Change of cheese volume during hot room ripening. - - strains $5,6,8,10,17,18$; strains 2, 3, 4, 7, 9, 14, 15, 16; - $-\boldsymbol{-}$-strain 12; -D-strains 1, 11, 13, CNRZ 85R, Control.

\section{Acide propionique}

La production d'acide propionique en cave chaude pour les différents groupes de souches figure dans le tableau III. Dans le cas de 12 souches $(2,4,5,6,8,9,10,14$, $15,16,17,18)$, les fromages ensemencés atteignent un palier de $800-900 \mathrm{mg} / 100 \mathrm{~g}$ de propionate dès 14 jours de cave chaude. Les analyses hebdomadaires, pratiquées sur les fromages fabriqués avec les souches 11 à 18 , montrent qu'au moins une de ces 12 souches (18) produit pratiquement cette quantité de propionate pendant la première semaine ( $642 \mathrm{mg} / 100 \mathrm{~g}$ à 7 jours). Inversement, la souche 16 ne se distingue pas des témoins à cette date : elle excrète donc la quasi-totalité de l'acide propionique durant la deuxième semaine. Les souches 14,15 et 16 présentent un comportement intermédiaire.

Trois souches $(3,7,11)$ produisent environ $800 \mathrm{mg} / 100 \mathrm{~g}$ d'acide propionique en 28 jours de cave chaude dont environ la 
Tableau III. Production de propionate en cours de cave chaude (mg/100 $\mathrm{g}$ de fromage). Propionate production during hot room ripening.

\begin{tabular}{|c|c|c|c|c|c|c|}
\hline \multirow[t]{2}{*}{ Souches } & \multicolumn{2}{|c|}{ Entrée cave chaude } & \multicolumn{2}{|c|}{14 jours cave chaude } & \multicolumn{2}{|c|}{28 jours cave chaude } \\
\hline & Moyenne & Écart type & Moyenne & Écart type & Moyenne & Écart type \\
\hline 1 & 23,4 & 17.0 & 795 & 117 & 892 & 124 \\
\hline ॥ & 8,4 & 0,4 & 485 & 99,5 & 854 & 180 \\
\hline III & 3,8 & 0,2 & 258 & 55,8 & 700 & 107 \\
\hline IV & 15,8 & 13,9 & 11,6 & 4,3 & 419 & 149 \\
\hline V & 0,7 & 0,6 & 4,8 & 5,6 & 9,5 & 6,0 \\
\hline Témoins & 4,1 & 2,3 & 13,3 & 12 & 26,6 & 18 \\
\hline
\end{tabular}

I : souches $2,4,5,6,8,9,10,14,15,16,17,18$; II : souches $3,7,11$; III : souches 12,13 ; IV : souche 1 ; V : souche CNRZ 85R.

I: strains 2, 4, 5, 6, 8, 9, 10, 14, 15, 16, 17, 18; II: strains 7, 11; III: strains 12, 13; IV: strain 1; V: strain CNRZ 85R.

moitié à 14 jours. L'activité de la souche 11 apparaît dès le septième jour et se poursuit lentement jusqu'à 21 jours. Deux souches $(12,13)$ produisent relativement peu d'acide propionique en 28 jours (respectivement 739 et $662 \mathrm{mg} / 100 \mathrm{~g}$ ), dont environ un tiers à 14 jours. La production finale de propionate par ces souches est inférieure $(P<$ $0,05)$ à celle de la souche la plus productrice (10).

La teneur en acide propionique des fromages ensemencés avec la souche 1 ne dépasse pas $400 \mathrm{mg} / 100 \mathrm{~g}$ en sortie de cave chaude. CNRZ 85R ne produit pas d'acide propionique, tout comme la flore résiduelle des témoins.

\section{Acide acétique}

Douze souches $(2,4,5,6,8,9,10,14,15$, $16,17,18$ ) produisent environ $350 \mathrm{mg} / 100 \mathrm{~g}$ d'acide acétique pendant les 2 premières semaines de cave chaude. Les souches $14,15,16,17$ et 18 excrètent l'acétate dans les mêmes conditions que le propionate. Cinq souches produisent ce niveau d'acétate après 28 jours uniquement. L'activité des souches 12 et 13 ralentit dès 21 jours.
Les souches 11 et CNRZ 85R n'excrètent pas plus d'acétate que les témoins : $50 \mathrm{mg} / 100 \mathrm{~g}$ environ (non montré).

\section{Lactates}

Quatorze souches $(2,3,4,5,6,8,9,10$, $11,14,15,16,17,18$ ) consomment plus de $75 \%$ des lactates pendant les 2 premières semaines de cave chaude (tableau IV). On retrouve encore les mêmes différences pour les souches ayant fait l'objet d'analyses hebdomadaires : disparition de $90-95 \%$ des lactates en 7 jours pour la souche 18 , entre le $7^{e}$ et le $14^{e}$ jour pour la souche 16 et un comportement intermédiaire pour les souches 14,15 et 17 . Trois souches $(7,12$, 13) utilisent environ $80 \%$ des lactates en 28 jours de cave chaude. La souche 1 métabolise environ la moitié des lactates disponibles pendant les 2 dernières semaines, tandis que CNRZ 85R ne consomme pas de lactate.

\section{Bilan des fermentations}

La confrontation de ces 4 indices par un classement des groupes de souches en 
Tableảu IV. Évolution de la teneur en lactates en cours de cave chaude. Change in lactate content of cheeses during hot room ripening.

\begin{tabular}{|c|c|c|c|c|c|c|}
\hline \multirow[t]{2}{*}{ Souches } & \multicolumn{2}{|c|}{ Entrée cave chaude } & \multicolumn{2}{|c|}{14 jours cave chaude } & \multicolumn{2}{|c|}{28 jours cave chaude } \\
\hline & Moyenne & Écart type & Moyenne & Écart type & Moyenne & Écart type \\
\hline A & 1313 & 172 & 145 & 135 & 68,6 & 83,2 \\
\hline B & 1342 & 61,5 & 880 & 247 & 268 & 152 \\
\hline C & 1288 & 188 & 1304 & 44,0 & 685 & 289 \\
\hline D & 1410 & 193 & 1354 & 34,0 & 1338 & 103 \\
\hline Témoins & 1355 & 170 & 1321 & 73,0 & 1278 & 65,5 \\
\hline
\end{tabular}

A : souches $2,3,4,5,6,8,9,10,11,14,15,16,17,18 ; B$ : souches $7,12,13 ; C$ : souche 1 ; D : souche CNRZ $85 R$. A : strains 2, 3, 4, 5, 6, 8, 9, 10, 11, 14, 15, 16, 17, 18. B: strains 7, 12, 13. C: strain 1. D: strain CNRZ 85R.

termes de précocité croissante révèle une bonne cohérence, même si la production d'acétate et la consommation des lactates sont peu discriminantes. Une certaine complémentarité de ces indices apparaît également, notamment entre les mesures de volume et les dosages d'acide propionique. La synthèse de ces données permet de distinguer 5 groupes de performances : 4 types de souches fromagères et un groupe de souches inaptes en technologie emmental.

Les souches les plus précoces, formant le premier groupe, $(5,6,8,10,17,18)$ achèvent leur fermentation propionique en 14 jours maximum de cave chaude (fig 2) : consommation des lactates, production de $\mathrm{CO}_{2}$, d'acétate et de propionate. Ce groupe est composé d'au moins deux sous-ensembles représentés par les souches 17 et 18 . La première semble produire des acides gras volatils en consommant les lactates progressivement durant toute cette période. La seconde conclut sa fermentation pratiquement après une semaine à $24^{\circ} \mathrm{C}$.

Un deuxième groupe $(2,4,9,14,15$, 16) se distingue du précédent par un dégagement massif de gaz plus tardif à 21 jours. Là encore, au moins 2 comportements différents coexistent : la fermentation de la souche 16 se déroule pendant la seconde semaine, celles des souches 14 et 15 apparaissent progressives pendant les 14 jours.

Les souches du troisième groupe $(3,7)$ terminent leur fermentation après 21 jours de cave chaude. Tous les traceurs (lactates, acétate, propionate, volume) l'indiquent.

Le profil de fermentation (fig 3 ) des souches du quatrième groupe (12) montre que leur fermentation s'achève uniquement

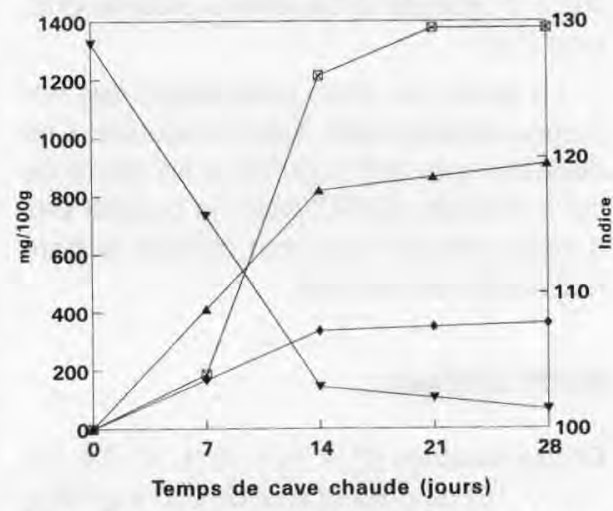

Fig 2. Profil de fermentation des souches du premier groupe. $-\square-$ volume (Indice) ; $-\mathbf{A}-$ propionate $(\mathrm{mg} / 100 \mathrm{~g})$; —- acetate $(\mathrm{mg} / 100 \mathrm{~g})$; - $\nabla$ - lactate $(\mathrm{mg} / 100 \mathrm{~g})$.

Fermentation profile of the first group strains. - - - volume (Indice); - $\mathbf{\Delta}-$ propionate $(\mathrm{mg} / 100 \mathrm{~g}) ; \longrightarrow$ - acetate $(\mathrm{mg} / 100 \mathrm{~g}) ;-\boldsymbol{\nabla}$ lactate $(\mathrm{mg} / 100 \mathrm{~g})$. 


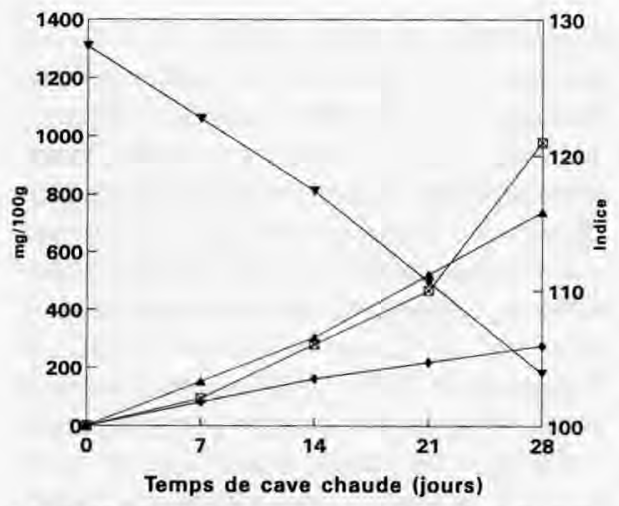

Fig 3. Profil de fermentation des souches du quatrième groupe. $-\square-$ volume (indice) ; $-\mathbf{A}-$ propionate $(\mathrm{mg} / 100 \mathrm{~g})$; - - acetate (mg/100 g) ; - - - lactate ( $\mathrm{mg} / 100 \mathrm{~g})$.

Fermentation profile of the fourth group strains. - - volume (indice); - $\mathbf{\Delta}$ - propionate ( $\mathrm{mg} / 100$ $\mathrm{g}) ;-\longrightarrow$ acetate (mg/100 g); - - lactate ( $\mathrm{mg} / 100 \mathrm{~g}$ ).

après 28 jours de cave chaude. Les concentrations finales en métabolites traduisent une fermentation peu poussée.

Le cinquième groupe $(1,11,13, \mathrm{CNRZ}$ 85R), hétéroclite, comprend les souches semblant inadaptées à la technologie emmental. CNRZ 85R présente un spectre plat : la composition et la conformation des fromages n'évoluent pas. La souche 1 se caractérise par une phase de latence très importante, les prémices d'une fermentation propionique n'apparaissant qu'après 4 semaines de cave chaude. Les souches 11 et 13 ont des cinétiques chimiques variables, mais ne produisent pas massivement de gaz carbonique.

\section{Vitesses de fermentation}

Malgré les grandes imperfections du mode de calcul des vitesses de fermentation (notamment à cause des intervalles importants entre les prélèvements susceptibles de provoquer des erreurs de détermination de la durée de fermentation), les résultats sont cohérents.
Les souches 11 et 13 ont des vitesses de production d'acides gras volatils faibles et proches : respectivement 37 et 29 $\mathrm{mg} / 100 \mathrm{~g} / \mathrm{j}$ de propionate et 16 et $12 \mathrm{mg} / 100$ $\mathrm{g} / \mathrm{j}$ d'acétate. Elles diffèrent cependant par leur temps de latence: pour la souche 11, 7 à 14 jours pour la consommation des lactates et la production d'acétate et 0 à 7 jours pour la production de propionate; pour la souche 13,7 à 14 jours pour l'évolution des lactates et de propionate, 14 à 21 jours pour la production d'acétate. La souche 12 présente des cinétiques équivalentes et des temps de latence intermédiaires. Ces souches apparaissent comme pas ou peu adaptées à la technologie de l'emmental si on considère l'ensemencement massif, la température élevée de cave chaude et la taille des fromages (où les fermentations sont souvent rapides et poussées).

Les souches 14,15 et 17 produisent des acides gras volatils de manière précoce à une vitesse moyenne. Leur temps de latence maximal est de 7 jours, les vitesses de fermentation de l'ordre de $55 \mathrm{mg} / 100 \mathrm{~g} / \mathrm{j}$ pour l'acide propionique, $20 \mathrm{mg} / 100 \mathrm{~g} / \mathrm{j}$ d'acide acétique et de 80 à $115 \mathrm{mg} / 100 \mathrm{~g} / \mathrm{j}$ de lactates. Ces différences de "rendement» suggèrent des voies métaboliques différentes et la production de composés non dosés.

La souche 16 présente un comportement inverse : temps de latence long ( 7 à 14 jours et 0 à 7 jours pour l'acétate) et vitesses élevées (40 mg/100 g/j d'acétate; $90 \mathrm{mg} / 100 \mathrm{~g} / \mathrm{j}$ de propionate; $135 \mathrm{mg} / 100 \mathrm{~g} / \mathrm{j}$ de lactates). Le temps de latence important est sans doute une conséquence directe de la grande sensibilité thermique de cette souche (perte de viabilité de 1,8 logarithme au cours du cycle thermique emmental).

La souche 18 allie un faible temps de latence ( 0 à 7 jours) et de fortes vitesses de fermentation ( $45 \mathrm{mg} / 100 \mathrm{~g} / \mathrm{j}$ d'acétate; $90 \mathrm{mg} / 100 \mathrm{~g} / \mathrm{j}$ de propionate et $150 \mathrm{mg} /$ $100 \mathrm{~g} / \mathrm{j}$ de lactates).

Le calcul de ces vitesses permet également d'apprécier la répétabilité de cette 
expérimentation en termes concrets. La concentration seuil, qui classe les souches dans les différents groupes, présente un écart type de $89 \mathrm{mg} / 100 \mathrm{~g}$. Cette valeur correspond à une journée ou une journée et demie pour les souches à forte $(90 \mathrm{mg} /$ $100 \mathrm{~g} / \mathrm{j})$ ou moyenne $(55 \mathrm{mg} / 100 \mathrm{~g} / \mathrm{j})$ vitesse de fermentation.

\section{DISCUSSION}

Au cours de cette expérimentation, la standardisation microbiologique et technologique était satisfaisante. L'épuration microbienne conduisait à l'obtention de laits ultrapropres dont les contaminations résiduelles étaient très faibles et proches de celles décrites par Trouvé et al (1991). La préparation des ferments propioniques était maîtrisée et conduisait à des ensemencements réguliers. Les paramètres de fabrication, les résultats intermédiaires et la composition globale des fromages étaient maîtrisés, répétables et conformes à la technologie de minifabrication des pâtes cuites (Buisson et al, 1987; Kerjean, 1991; Riberolles et Quiblier, 1991). Cette étude se distinguait des précédentes par l'emploi de lait ultrapropre dont la faible contamination résiduelle, notamment propionique, permettait d'observer sans ambiguité le comportement des souches introduites.

La population ensemencée dans cette étude est plus élevée que dans les travaux précédents : d'un facteur 10 (Turner et al, 1983 ; Crow et Turner, 1986 ; Pérréard, 1992) à 100 (Baer et al, 1993). Cette inoculation massive, associée à une température de cave chaude plus élevée d'environ 2 degrés, permet d'éliminer les souches ne montrant pas d'aptitude technologique manifeste dans ces conditions favorables.

Les profils de fermentation enregistrés s'apparentent à ceux décrits par Turner et al (1983), Crow et Turner (1986) ou Steffen et al (1993). Les conditions différentes d'épuration ou d'ensemencement du lait (lactique et propionique) et d'affinage des fromages ne permettent pas de comparaison plus poussée. Dans notre étude, l'analyse des fermentations montre que les productions d'acide propionique et de gaz carbonique sont les indices les plus discriminants. Cependant, les voies métaboliques décrites par Crow et Turner (1986) et Sebastiani et Bader (1992) suggèrent que la consommation des lactates et la production d'acétate constituent également de bons traceurs. Ils interviennent à 2 niveaux : quantitatif, en décrivant l'intensité de la fermentation, et qualitatif, en déterminant la (les) voie(s) métabolique(s) utilisée(s). Par exemple, une moindre consommation des lactates associée à de fortes productions de $\mathrm{CO}_{2}$ et d'acétate peut révéler une forte activité de désamination de l'aspartate (Crow et Turner, 1986).

L'analyse hebdomadaire des fromages fournit des informations plus fines sur le comportement des souches propioniques. Ces prélèvements autorisent l'établissement de profils de fermentation beaucoup plus précis et le calcul de temps de latence et de vitesses de production de métabolites. Ces éléments permettent de discriminer des souches classées dans un même groupe suivant le protocole de la première série. De plus, des éléments qualitatifs tels que les vitesses de fermentation jouent sur la qualité de l'ouverture : nombre et taille des yeux notamment. Ces vitesses de fermentation sont relativement élevées par rapport à celles enregistrées par Turner et al (1983), Crow et Turner (1986) ou Thierry et al (1994) : 10 à $30 \mathrm{mg} / 100 \mathrm{~g} / \mathrm{j}$ d'acétate et 15 à $60 \mathrm{mg} / 100 \mathrm{~g} / \mathrm{j}$ de propionate. La différence de température de cave chaude explique en partie ces résultats.

Les mesures du volume des fromages présentent quelques limites en raison de la diffusion du gaz à travers la paraffine et surtout de l'éclatement de cette dernière après la phase d'expansion. Les résultats obte- 
nus sont donc purement qualitatifs : seul le délai de production massive de gaz, se traduisant par une différence significative de volume, peut être rigoureusement pris en compte. Dans cette étude, les mesures de volume discriminent les souches des premier et deuxième groupes, aux cinétiques chimiques équivalentes. Elles classent aussi les souches 11 et 13 dans le cinquième groupe des bactéries inaptes à l'ouverture en technologie emmental, alors que leur production d'acides gras volatils est relativement importante.

On pourrait penser que ces 2 souches particulières utilisent en grande partie la voie métabolique de fixation du $\mathrm{CO}_{2}$ sur le propionate (Crow et Turner, 1986). Globalement, le manque de cohérence des rapports $\mathrm{C}_{3} / \mathrm{C}_{2}$ produits ne permet pas de confirmer cette hypothèse. Au contraire, en ne considérant que la deuxième série, ce ratio est significativement plus élevé pour cette souche : 3,48 contre 2,59 pour un groupe de 5 souches $(12,13,15,17,18)$, les souches 14 et 16 présentant des valeurs intermédiaires. On peut noter qu'un rapport de 2,59 correspond approximativement à l'équation de Fitz : rapport molaire de 2 , rapport massique de 2,47. L'observation de ratios $C_{3} / C_{2}$ supérieurs à cette valeur suggère l'existence de voies métaboliques originales, puisque l'équation de Fitz produit plus d'acide propionique que les autres voies métaboliques décrites jusqu'à présent. Crow et Turner (1986) suggéraient ainsi une utilisation possible des citrates à partir du cycle de Krebs conduisant à une forte production de propionate.

Les bilans carbonés (acétate + propionate $+\mathrm{CO}_{2}$ divisés par les lactates consommés) varient entre $86,8 \%$ et $117,6 \%$ suivant les souches testées dans la deuxième série, la quantité de gaz carbonique produite étant considérée comme équimolaire à celle de l'acétate pour les 3 voies métaboliques connues. Or l'incertitude globale de ces dosages s'élève à $5,7 \%$ en prenant en compte les valeurs relatives de $3 \%$ pour les lactates (Steffen, 1971) et $8 \%$ pour les acides gras volatils (Berdagué, 1986). La production de succinate et la consommation d'aspartate (et/ou de propionate) peuvent expliquer ces différences de bilans. Selon Crow et Turner (1986), les dosages de succinate et d'aspartate permettent également de déterminer les voies métaboliques utilisées par les différentes souches. Ces voies métaboliques conditionnent le rendement en gaz carbonique de la fermentation. Ces dosages n'ont pas été effectués au cours de notre étude, mais aucune différence significative de teneur en ammoniaque (issue de la désamination de l'aspartate) n'a été enregistrée. II apparaît donc difficile de se prononcer sur d'éventuelles différences métaboliques, même si, empiriquement, elles apparaissent très probables.

Globalement, cette expérimentation classe les 19 souches testées en 5 grands groupes : 4 se composent de souches aptes à la technologie de l'emmental (c'est-à-dire développant une forte production gazeuse pendant l'affinage) à des degrés divers, le dernier comporte les souches inaptes en technologie emmental. De plus, les 2 premiers groupes contiennent au moins 2 sousensembles chacun, représentés respectivement par les souches 17 et 18,16 et 14/15. La confrontation des performances des souches 1 à 5 avec celles enregistrées en fermenteur (Trossat, 1992) et en fromagerie expérimentale sur lait bactofugé (Pérréard, 1992) est cohérente. Ces méthodes classent de manière identique 4 souches sur 5 dans 3 catégories : lente, intermédiaire et rapide. La minifabrication semble donc être un bon outil de criblage des souches de bactéries propioniques, plus ou moins poussé selon le protocole utilisé : cinétique chimique hebdomadaire ou bimensuelle. Cette classification permettra à chaque utilisateur de choisir les souches qui conviennent à sa technologie particulière de fabrication et d'affinage, en syn- 
chronisant l'évolution rhéologique de la pâte et la production de gaz carbonique et de substances aromatiques notamment. Le fromager pourra également sélectionner des souches plutôt productrices de gaz ou d'acides gras volatils. Par exemple, après 14 jours de cave chaude, la souche 5 (du premier groupe) produit approximativement $800 \mathrm{mg} / 100 \mathrm{~g}$ de propionate et provoque une augmentation de $24 \%$ du volume des fromages ; la souche 4 (du deuxième groupe) accroît ce volume de $12 \%$ pour une production équivalente d'acides gras volatils.

De nombreux auteurs (Langsrud et Reinbold, 1973 ; Desmazeaud et al, 1974 ; Perez Chaia et al, 1987 ; Zehntner, 1993 ; Sollberger, 1994) ont montré de fortes interactions microbiennes entre les bactéries lactiques et propioniques. La stimulation ou l'inhibition des bactéries propioniques semble très dépendante de la souche, ce qui implique que les aptitudes technologiques enregistrées ici peuvent être tributaires des souches de ferments lactiques utilisés, voire de la nature des contaminants. Dans un fromage industriel, fabriqué avec du lait peu épuré, le comportement des souches propioniques peut également différer à cause d'interactions avec la flore lactique sauvage du lait et des contaminations en cours de fabrication.

Quoi qu'il en soit, ces résultats représentent l'aptitude technologique des souches dans les conditions standardisées de la minifabrication. Ils doivent être confirmés en fromagerie expérimentale à partir de lait microfiltré. Les différences de comportement observées feront l'objet d'études de laboratoire plus fondamentales sur le métabolisme des bactéries propioniques. Cela montre bien le rôle de la minifabrication comme interface entre les expérimentations fondamentales et fromagères.

L'influence de différents facteurs technologiques (report de longue durée à basse température, teneur en sel des fromages, conservation après affinage, interaction lac- tobacilles/propionibactéries, etc) est actuellement à l'étude. Ces résultats, comparés à ceux présentés ici permettront aux utilisateurs fromagers de choisir les souches adaptées à leur technologie particulière.

\section{REMERCIEMENTS}

Les auteurs tiennent à remercier vivement toute l'équipe du laboratoire central de l'Institut technique du gruyère à Rioz pour les analyses sur les fromages, le Laboratoire de recherches de technologie laitière (INRA) de Rennes pour la mise à disposition du matériel de traitement du lait et d'analyse microbiologique, et particulièrement $P$ Boyaval, $S$ Lortal et $A$ Thierry pour la relecture de ce document.

\section{RÉFÉRENCES}

Baer A, Ryba I, Grand M (1993) Ursachen der Entstehung von braunen Tupfen im Kâse. Schweiz Milchwirtsch Forsch 22, 3-7

Berdagué Jl. (1986) Méthode d'extraction rapide des acides gras volatils dans les fromages. Lait 66, 233246

Buisson V, Kerjean JR, Courroye M (1987) Miniaturisation de la fabrication fromagère. Tech Lait Market $1024,17-23$

CNERNA (1986) Recommandations pour l'estimation de la contamination du lait en spores de clostridia. Rev Lait Fr 451, 39-45

Collin JC, Berdagué JL, Dognin-Bergeret M, Grappin R (1987) Affinage et qualité du gruyère de Comté. IV. Étude de la protéolyse. Lait 67, 229-318

Collomb M, Spahni M, Badertscher R (1992) Optimisation des conditions d'entreposage des échantillons de fromage en laboratoire à l'aide d'analyses chimiques. Schweiz Milchwirtsch Forsch 21, 6-11

Courroye M, Berdagué JL, Leray O (1989) Application de la technique FIA (Flow Injection Analysis) au dosage des fractions azotées du fromage. Lait 69, 233-241

Crow V, Turner KW (1986) The effect of succinate production on other fermentation products in Swiss-type cheese. N Z J Dairy Sci Technol 21, 217-227

Desmazeaud M, Vassal L, Devoyod JJ (1974) Rôle des substances peptidiques stimulant la production de gaz par les bactéries propioniques au cours de l'affinage du fromage de gruyère et d'emmental. $X I X^{e}$ Congr Int Lait New Delhi, B6, 462-463

Dupuis C, Boyaval P (1993) Esterase activity of dairy Propionibacterium. Lait 73, 345-356 
Dupuis C, Corre C, Boyaval P (1993) Lipase and esterase activities of Propionibacterium freudenreichii subsp freudenreichii. Appl Environ Microbiol 59, 4004-4009

El Soda M. Ziada N, Ezzat N (1992) The intracellular peptide-hydrolase system of Propionibacterium. Microbios 72, 65-74

FIL/DF (1982) Fromages et fromages fondus. Determination de l'extrait sec total; méthode de référence. Norme FIL 4A, Bruxelles, Belgique

FIL/IDF (1991a) Lait et produits laitiers. Dénombrement des micro-organismes. Comptage des colonies à $30^{\circ} \mathrm{C}$. Norme FIL 100B, Bruxelles, Belgique

FILIDF (1991b) Lait et produits laitiers. Détermination de la teneur en matière grasse. Guide de directives générales appliquées aux méthodes butyrométriques. Norme FIL 152, Bruxelles, Belgique

Fryer H, Peberdy MF(1977) Growth of Propionibactera in Swiss cheese and Egmont cheese. $N Z J$ Dairy Sci Technol 12, 33-34

Gautier M, Mouchel N, Rouault A, Sanseau P (1992a) Determination of genome size of four Propionibacterium species by pulsed field gel electrophoresis. Lait 72, 421-426

Gautier M, Rouault A, Lortal S, Leloir Y, Patureau D (1992b) Characterization of a phage infecting Propionibacterium freudenreichii. Lait 72, 431-435

Gouet JP. Phillipeau G (1989) Comment interpréter les résultats d'une analyse de variance ? Institut technique des céréales et des fourrages. ITCF, Paris, France

Heiss (1961) Essais de dosage de la matière grasse dans le fromage par des méthodes rapides. Dtsch Molk Ztg 82, 67-70

Hettinga DH, Reinbold GW (1972) The propionic acid bacteria - A review. I. Growth. J Milk Food Technol 35, 295-301

Hollywood NW, Doelle HW (1984) The effect of sampling position and age on levels of propionibacteria and flavour related compounds in Swiss-type cheese. Aust J Dairy Technol 39, 80-82

Kerjean JR (1991) Minifabrication de fromages à pãte pressée cuite : les choix de IITG depuis 1987. Colloque INRAVENSAR, Qualité des laits à la production et aptitudes fromagères, 23-24 janvier, Rennes, France

Langsrud T, Reinbold GW (1973) Flavour development and microbiology of Swiss cheese. A review. III. Ripening and flavor production. J Milk Food Technol 36, 593-609

Lemée R, Rouault A, Guezenec S, Lortal S (1994) Autolysis of fitty seven strains of dairy propionibacteria. Lait $74,241-251$

Naud Al, Legault-Demarre J, Ryter A (1988) Induction of a stable morphological change in Propionibacterium freudenreichii. J Gen Microbiol 134, 283-293

Perez-Chaia A, Pesce de Ruiz Holgado A, Oliver G (1987) Interaction between Lactobacillus helveticus and Propionibacterium freudenreichii subsp. shermanii. Microbiol Aliments Nutr 5, 325-331
Pérréard E (1992) Bactéries propioniques : essais de validation fromagère des tests en fermenteurs. Parlons Technologie 6, 17-18. ITG, Rennes, France

Phillipeau G (1984) Puissance d'une expérience. Institut technique des céréales et des fourrages. ITCF, Paris, France

Reinbold GW (1972) Swiss cheese varieties. Pfizer cheese monographs, vol 5. Pfizer Inc, New York, USA

Riberolles A, Quiblier JP (1991) Du lait microfiltré pour l'emmental. Parlons Technologie 5, 6-8. ITG, Rennes, France

Sahlstrom S, Espinosa C, Langsrud T, Sorhaug T(1989) Cell wall, membrane and intracellular activities. J Dairy Sci 72, 342-350

Samples DR, Richter RL, Dill CW (1984) Measuring proteolysis in cheddar cheese slurries : comparison of Hull and trinitrobenzene sulfonic acid procedures. J Dairy Sci 67, 60-63

Sebastiani H, Bader T (1992) Métabolisme des bactéries propioniques. Milchwirtschaftliche Berichte aus den Bundesanstalten Wolfpassing und Rotholz 112, 137 140

Sherman JM (1921) The cause of eyes and characteristic flavor in Emmental or Swiss cheese. J Bacterio/ 6 , 379-392

Sollberger H (1994) Parameter zur Frūherfassung des Nachgärungsrisikos beim Emmentaler-Käse. Schweiz Milchwirtsch Forsch 23, 20-24

Steffen C (1971) Methoden zur Bestimmung der Gesamtmilchsäure und der Lactatkonfiguration in Käse und Milch. Schweiz Milchztg 82, 1073-1078

Steffen C, Eberhard P, Bosset JO, Rüegg M (1993) Swiss varieties. In: Cheese : chemistry, physics and microbiology, vol 2, 2nd edn (Fox PF, ed). Elsevier Applied Science, London

Thierry A, Madec MN, Richoux R (1994) Croissance des bactéries propioniques dans le fromage : comparaison de deux milieux de dénombrement. Lait $74,161-171$

Trossat P (1992) Test des bactéries propioniques en fermenteur: la méthode ITG. Parlons Technologie 6, 15-16. ITG, Rennes, France

Trouvé E, Maubois JL, Piot M, Madec MN, Fauquant J, Tabard J, Brinkman G (1991) Rétention de différentes espèces microbiennes lors de l'épuration du lait par microfiltration en flux tangentiel. Lait 71, 1-13

Turner KW, Morris HA, Martley FG (1983) Swiss-type cheese. II. The role of thermophilic lactobacilli in sugar fermentation. NZJ Dainy Sci Technol 18, 117-123

Vassal L, Auclair J (1974) Aptitude de différentes souches de bactéries propioniques à la formation de l'ouverture dans le fromage de gruyère. Bréves Commun XIXe Congr Int Lait, New Delhi, 796

Zehntner U (1993) Influence des bactéries lactiques sur la multiplication des bactéries propioniques. Rapport d'activité 1992 de la Station fédérale de recherches laitières, Liebefeld/Beme, Suisse, 217-219 